\title{
Research on the Application of Internet of Things and VR Technology in the Protection and Development of Shouzhou Kiln
}

\author{
Xuelian Yu $\mathbb{D}^{1,2}$ and Yajun Zhang $\mathbb{D}^{2,3}$ \\ ${ }^{1}$ Graduate School, University of Perpetual Help System DALTA, Philippines \\ ${ }^{2}$ School of Art, Anhui University of Finance and Economics, Bengbu, China \\ ${ }^{3}$ Faculty of Design and Architecture, University Putra Malaysia, Malaysia \\ Correspondence should be addressed to Yajun Zhang; 120081521@aufe.edu.cn
}

Received 30 September 2021; Accepted 3 November 2021; Published 18 November 2021

Academic Editor: Deepak Gupta

Copyright (c) 2021 Xuelian Yu and Yajun Zhang. This is an open access article distributed under the Creative Commons Attribution License, which permits unrestricted use, distribution, and reproduction in any medium, provided the original work is properly cited.

\begin{abstract}
The Internet of things (IoT) has changed the information transmission mode of people, things, and services. The new functional experience will bring users an efficient, convenient, and safe lifestyle. Virtual reality (VR) technology, also known as spiritual environment technology, is a new stage of the development of computer and information technology. It is an important auxiliary tool in the fields of $\mathrm{R} \& \mathrm{D}$, production, education, and so on. As one of the six most famous kilns in the Tang Dynasty (618-907 BC), Shouzhou kiln plays an important role in the history of ceramics in China and even in the world. However, due to the long history, incomplete site preservation, fragile ceramic materials, and lack of effective promotion and publicity, it has been difficult to make a breakthrough in the protection and development of Shouzhou kiln. VR technology makes comprehensive use of computer software and hardware, breaks the barrier between virtual and reality, and provides a new idea for the protection and development of Shouzhou kiln. On the basis of fully combing the principle of VR technology and analyzing the application possibility of VR technology, firstly, focusing on the history and from the perspective of archaeological research of Shouzhou kiln, this paper discusses how to use VR technology to realize the restoration of ancient kiln sites, ancient ceramics, ancient ceramics technology, and relevant data preservation of Shouzhou kiln. Secondly, focusing on the present, this paper discusses the application of VR technology and Internet of things technology in Shouzhou kiln ceramic product design, Shouzhou kiln process technology improvement, Shouzhou kiln ceramic product display, and Shouzhou kiln marketing. Finally, focusing on the future, this paper discusses the application prospect of VR technology in the inheritance of Shouzhou kiln technology and the promotion of Shouzhou kiln ceramic culture. It is expected to explore feasible paths and methods for the protection, inheritance, and development of ancient ceramic culture in China and even the world through the discussion of VR technology involved in the protection and development of Shouzhou kiln.
\end{abstract}

\section{Introduction}

The Internet of things (IoT) is realized and developed on the basis of the Internet, telecommunication network, and radio and television network. It is an extension and expansion on the basis of three networks. User application terminals have expanded from information interaction and communication between people to communication and connection between people and things, things and things, and things and people. Therefore, the Internet of things technology can make objects more intelligent. It has increasingly become a new point of technological innovation and economic growth. Many American colleges and universities, such as the University of California, Berkeley, Massachusetts Institute of Technology, and Auburn University, and well known enterprises such as, IBM, Crossbow Technology, have put forward relevant solutions for the Internet of things technology. The U.S. government policies to encourage the development of the Internet of things technology are mainly reflected in energy, broadband, and healthcare three areas of medical treatment. In August 2009, the Japanese government upgraded the "U-Japan" plan to the "I-Japan" strategy 2004, 
which is committed to building an intelligent Internet of things service system. Meanwhile, countries such as Australia, Singapore, South Korea, France, Germany, and Australia are also stepping up the deployment of the Internet of things technology. Many countries are gradually promoting construction of the next-generation ubiquitous network infrastructure.

Virtual reality technology is a new stage in the development of computer and information technology. It refers to "in the computer software and hardware, various sensors, graphics and image generation systems, as well as special clothing, three-dimensional environment with certain audio-visual touch and smell, users can communicate with this computer-generated virtual reality in a concise and natural way with the support of these software and hardware equipment" interact with objects in the real world. It is the result of the comprehensive integration of modern highperformance computer system, artificial intelligence, computer graphics, man-machine interface, three-dimensional image, three-dimensional sound, measurement control, simulation, and other technologies. The purpose is to establish a more harmonious artificial environment [1].

In the 1960s and 1970s, VR technology was in the brewing stage of ideas, concepts, and technologies. In 1965, Dr. Suther [2], an important founder of computer graphics, published a short article "the ultimate display", describing a new display technology. Users were directly immersed in the virtual environment controlled by computers. At the same time, users can also interact with objects in the virtual environment. In the 1980s, with the development of personal computer technology and computer network, the development of VR technology accelerated. During this period, the VR system with practical value appeared and a relatively complete reality simulation was realized through a VR helmet. In 1987, Foley published "interfaces for advanced computing" in Scientific American magazine. The magazine also published articles on data gloves, which attracted people's attention. In 1989, Lanier, the founder of the American VPL company, first proposed the word "virtual reality," which became a special name in this field of science and technology. After the 1990s, with the breakthrough and rapid development of computer technology and highperformance computing, computer network and communication, human-computer interaction equipment, and other scientific and technological fields, as well as the huge demand in application fields, VR technology has entered a period of rapid development. In 1993, Heim [3] proposed that "VR is an event or entity in effect rather than in fact" and described the seven characteristics of VR: simulation, interaction, artificial reality, immersion, telepresence, whole-body immersion, and network communication. In 1994, Burdea et al. [4] published "Virtual Reality Technology," in which they summarized the basic characteristics of VR with 3I (immersion, interaction, and imagination). The software platform and modeling language for VR system development are developed. Quantum 3D developed open GVS in 1989 and sensors launched WTK in 1992. VRML was first proposed at the first WWW conference held in Geneva in March 1994, and the formulation of relevant international standards began. In 2005, ITU formally defined the concept of the "Internet of things" at the World Summit on the Information Society (WSIS) held in Tunis and then issued "ITU Internet Reports 2005-the Internet Things," which introduced the characteristics, related technologies, challenges, and future market opportunities of the Internet of things, pointing out the direction for the rapid development of the Internet of things. The Internet of things enables us to obtain a new communication dimension in the world of information and communication technology, and also enables the virtual world of VR technology to finally become a reality.

China's research on VR technology began in the 1970s, when it was mainly concentrated in the field of aerospace. In the early 1990s, some Chinese universities and scientific research institutes began to study VR from different angles. In 1996, Chengcheng et al. of Tsinghua University and other three people published China's first book on VR, "The Theory, Implementation and Application of Spiritual Environment (Virtual Reality) Technology" [5]. In the same year, Qinping and others published the overview of VR research [6]. The ministry of science and technology of the People's Republic of China and the National Natural Science Foundation of China began to give key grants to the research in the field of VR. The national " 863 " plan identified the "distributed virtual environment" as a key project in 1996 and implemented the DVENET plan. With the maturity of China's technology and theory in the field of VR, after 2010, its discussion scope has rapidly expanded to the fields of ancient ceramic protection, ceramic product design, and ceramic culture inheritance in China. In 2012, Cheng and others proposed VR-based modeling and reverse restoration technology of ancient ceramic relics. In 2014, Hu analyzed the feasibility of VR technology to support ancient ceramic cultural relics. Taking Jingdezhen ancient kiln as an example, this paper discusses the use of VR technology to display ancient ceramic sites, so as to promote the inheritance and dissemination of the ceramic culture [7]. In terms of ceramic product design and technology, He discussed the role of virtual reality in the traditional nonrelic firing technology of Jun porcelain in his paper "Innovative Exploration of VR Ceramic Firing Simulation Technology" in 2019. In 2021, Wang summarized the value of VR interactive technology in ceramic cultural and creative product design in his paper.

\section{Application of VR Technology in Ancient Ceramic Restoration, Ancient Kiln Site Restoration, and Related Data Preservation of Shouzhou Kiln}

In terms of cultural heritage protection at home and abroad, with the help of VR technology and digital synthetic image and other technical frameworks, the cultural heritage and sites are digitally copied and simulated and the simulated reproduction of cultural relics is realized by digital means. The core of virtual reality technology is modeling and simulation. Digital technology is used to effectively preserve cultural relics, realize the three-dimensional or model establishment of data, and scientifically, effectively, accurately, 
and permanently preserve endangered cultural relics. [8] Using VR technology for restoration and reproduction can shorten the restoration period of Shouzhou kiln cultural relics, establish an online virtual museum through computer network, and provide the public with novel and intuitive Shouzhou kiln cultural relic resources. In addition to the above points, the role of VR technology in Shouzhou kiln archaeology is also reflected in the sensory impact. If VR technology can be applied during the exhibition of ancient ceramic relics of Shouzhou kiln, the exhibition environment of ancient ceramic relics will become more open and intuitive. In this way, it can be ensured that after the ancient ceramic relics of Shouzhou kiln are displayed by using VR technology, visitors can more clearly and intuitively contact the relics and feel the strong impact of different aspects such as vision and perception.

In terms of specific application, VR reverse engineering technology can be used to restore the fragments of ceramic relics of Shouzhou kiln and the Shouzhou kiln site. The methods are briefly introduced as follows:

Step 1. Measure and scan the existing ceramic relics, fragments, or ancient kiln sites of Shouzhou kiln with CMM and $3 \mathrm{D}$ laser scanner to obtain their data point clouds and align them quickly.

Step 2. Use data optimization software such as surface to analyze results, optimize data points, remove useless information, and quickly extract product feature point cloud.

Step 3. According to the convenience of drawing, intercept the profile of the restored object in an all-round way, draw the product surface by using 3D CAD software such as Pro/E and UG, and complete the virtual reconstruction of $3 \mathrm{D}$ product modeling.

Step 4. The surface can be repaired and restored by software according to the condition of the surface, and then, it can be output into an STL format file, which can be processed and manufactured by connecting the ceramic 3D printer and other processing equipment, or use an ordinary $3 \mathrm{D}$ printer to print it out and make the mold after equal scale amplification. Alternatively, it can be exported to STL or IGES format files, imported into 3DMAX or rhino for rendering and $3 \mathrm{D}$ data saving, or transferred to Virtools and Cult3D software for interactive virtual demonstration, as shown in Figure 1.

\section{Application of VR Technology in Shouzhou Kiln Ceramic Product Design, Shouzhou Kiln Process Technology Improvement, Shouzhou Kiln Ceramic Product Display, and Shouzhou Kiln Marketing}

3.1. VR Technology Involved in the Design and $R$ \& $D$ of Shouzhou Kiln Ceramic Products. VR technology can convey the design scheme of Shouzhou kiln products through virtual reality and comprehensively check the shape and color of product design with the help of relevant hardware, VR glasses, data pen, etc. Through simulated firing, the possible defects of Shouzhou kiln products in the firing process can be further reduced. VR technology not only strengthens the interactive virtual experience of designers when observing products, so that they can more effectively feel the performance of products. It can also copy, repair, and save data of existing products. It can also be used as a performance testing tool for Shouzhou kiln products for allround evaluation and correction before production. Using VR technology to realize the virtual detection and evaluation of Shouzhou kiln ceramic products can further simulate its production process and build a virtual detection and evaluation system, so that its quality can be monitored and guaranteed more effectively before it is officially put into production.

The specific method is to use the modeling software to construct the digital three-dimensional modeling and paste the material. Through the signal transformed from Virtools into virtual reality, the designer can modify the design draft through virtual reality hardware and then feedback the modified data to the modeling or color texture stage to improve the product. Finally, the detection module is used to detect, analyze whether the hidden dangers and defects are within the allowable error range, and then make necessary corrections, so as to effectively ensure the quality requirements of the product and improve the efficiency. See Figure 2 for the specific process.

3.2. VR Technology Involved in Raw Material Improvement of Shouzhou Kiln. Shouzhou kiln has a long history of using materials nearby as tire and glaze of ceramic products. As early as the Northern and Southern dynasties (420 589), the clay of Shangyao town in Huainan was developed and utilized for manufacturing Shouzhou porcelain. According to the records of Huaiyuan County annals, "sixty miles to the south of the county, near the Luohe River, there are two winding peaks, under which there are coal kilns, Aboriginal stone burning ash, Bo mud filling, burning basin, and other utensils. Red chalk soil is also produced, and the glaze is taken by those who paint it" [9]. Until the beginning of the Ming dynasty (1368-1644), Shangyao people used the clay and mountain glaze from crow mountain, cave mountain, Wu family memorial archway, and other places to burn pottery jars and other pottery products. In the late 1950s, clay was found in Datong, Dongshan, Bagongshan, and other places, which was later developed and utilized by Datong bowl factory, municipal ceramic factory, and Bagongshan refractory factory. In the early 1960s, minerals such as clay, feldspar, and purple shale were found in Xiejiaji, Bagongshan, and Shangyao, respectively, which were developed and utilized by Huainan porcelain factory, Fengtai pot and bowl factory, and Shangyao cylinder factory.

The element composition of ancient Shouzhou kiln porcelain was determined by energy-dispersive X-ray fluorescence (EDXRF) technology. The results are shown in Table 1.

The results of the determination of the composition of the tire glaze of Shouzhou kiln ceramic products are as follows: the main components of the tire are silicon dioxide, aluminum oxide, and iron oxide. The glaze is mainly composed of silicon dioxide, aluminum oxide, calcium oxide, and phosphorus pentoxide. There are two groups of data 


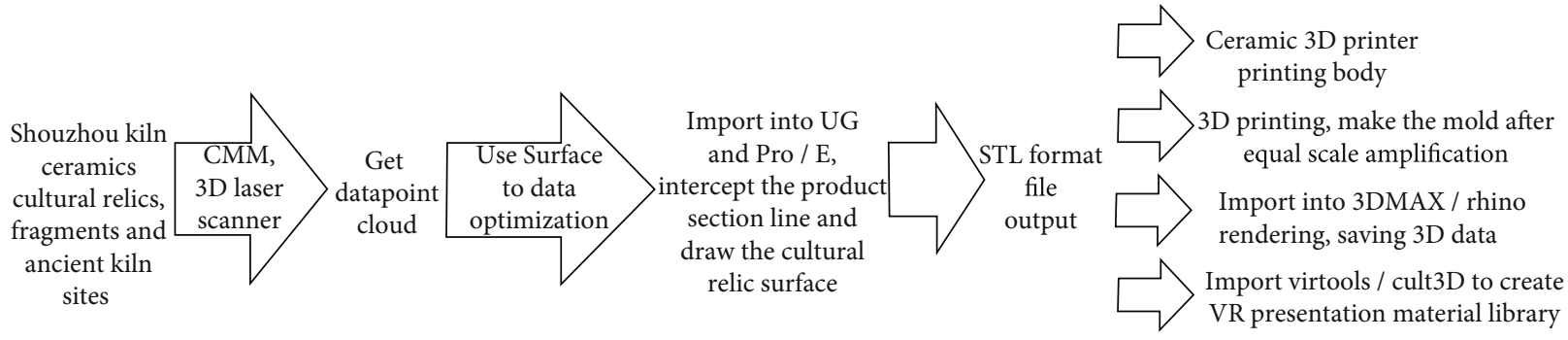

Figure 1: Reproduction, restoration, and data preservation of ceramic relics in Shouzhou kiln.

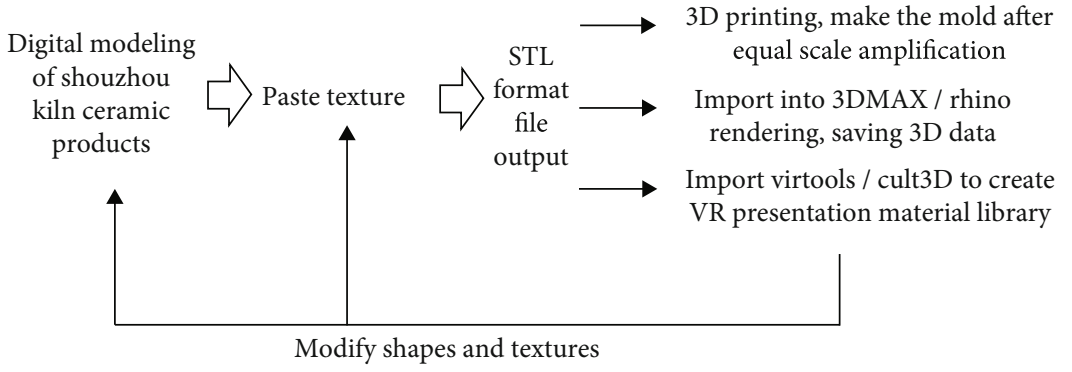

FIGURE 2: Flow chart of VR intervention in Shouzhou kiln ceramic product design.

TABle 1: Chemical composition analysis of ancient Shouzhou kiln fragments [10].

\begin{tabular}{|c|c|c|c|c|c|c|c|c|c|c|c|c|}
\hline Kiln site & Sample & & $\mathrm{SiO}_{2}$ & $\mathrm{Al}_{2} \mathrm{O}_{3}$ & $\mathrm{Fe}_{2} \mathrm{O}_{3}$ & $\mathrm{TiO}_{2}$ & $\mathrm{CaO}$ & $\mathrm{MgO}$ & $\mathrm{K}_{2} \mathrm{O}$ & $\mathrm{Na}_{2} \mathrm{O}$ & $\mathrm{P}_{2} \mathrm{O}_{5}$ & $\mathrm{MnO}_{2}$ \\
\hline \multirow[t]{2}{*}{ Guanzuizi } & Grayish-brown buttress & & 56.11 & 20.33 & 4.58 & 2.73 & 0.95 & 4.95 & 2.77 & 2.25 & 0.05 & 0.11 \\
\hline & & Body & 56.06 & 25.05 & 3.51 & 1.29 & 0.78 & 4.94 & 2.01 & 2.99 & 0.14 & 0.037 \\
\hline \multirow[t]{3}{*}{ Guanzuizi } & Blue-glaze & Engobe & 56.39 & 23.98 & 3.09 & 1.23 & 1.73 & 4.29 & 4.04 & 1.83 & 0.14 & 0.04 \\
\hline & & Glaze & 45.89 & 15.38 & 2.81 & 0.85 & 19.46 & 4.9 & 3.74 & 1.06 & 1.4 & 0.54 \\
\hline & & Body & 55.54 & 25.49 & 3.4 & 1.27 & 0.88 & 5.01 & 1.91 & 3.43 & 0.087 & 0.047 \\
\hline \multirow[t]{3}{*}{ Guanzuizi } & Blue-glazed porcelain & Engobe & 54.38 & 21.9 & 2.4 & 0.92 & 5.48 & 5.23 & 3.48 & 2.66 & 0.33 & 0.77 \\
\hline & & Glaze & 46.49 & 13.69 & 3.88 & 0.81 & 20.44 & 4.95 & 2.6 & 1.22 & 1.18 & 0.42 \\
\hline & & & 54.66 & 26.69 & 2.69 & 1.19 & 0.91 & 4.97 & 1.25 & 4.36 & 0.23 & 0.057 \\
\hline \multirow[t]{2}{*}{ Guanzuizi } & Blue-glazed porcelain & Engobe & 55.43 & 23.93 & 2.11 & 0.94 & 1.37 & 5.06 & 4.16 & 3.97 & 0.17 & 0.043 \\
\hline & & & 47.4 & 12.6 & 2.38 & 0.73 & 19.87 & 5.38 & 4.01 & 1.43 & 1.26 & 0.42 \\
\hline \multirow[t]{2}{*}{ Inpatient department } & Gray buttress & & 57.03 & 23.61 & 3.33 & 1.43 & 1.11 & 4.9 & 2.32 & 3.06 & 0.18 & 0.06 \\
\hline & & Douy & 59.37 & 20.37 & 4.7 & 0.96 & 1.06 & 4.8 & 2.54 & 2.64 & 0.18 & 0.083 \\
\hline \multirow[t]{2}{*}{ Inpatient department } & Yellow-glazed porcelain & Engobe & 60.31 & 19.73 & 2.67 & 1.03 & 1.21 & 5.35 & 2.63 & 3.31 & 0.22 & 0.063 \\
\hline & & Glaze & 48.88 & 11.94 & 3.8 & 0.77 & 17.92 & 5.07 & 4.35 & 1.57 & 1.47 & 0.52 \\
\hline \multirow{3}{*}{ Inpatient department } & & Body & 57.24 & 21.25 & 4.53 & 1 & 0.87 & 5.19 & 2.78 & 3.4 & 0.37 & 0.093 \\
\hline & Yellow-glazed porcelain & Engobe & 62.24 & 18.75 & 3.24 & 1.21 & 0.84 & 4.98 & 2.87 & 3.1 & 0.18 & 0.037 \\
\hline & & Body & 57.86 & 20.39 & 5.25 & 1.02 & 1.25 & 4.8 & 2.93 & 2.89 & 0.11 & 0.083 \\
\hline \multirow[t]{2}{*}{ Inpatient department } & Yellow porcelain & Engobe & 63.36 & 16.81 & 3.36 & 0.67 & 1.73 & 5.56 & 2.22 & 2.51 & 0.1 & 0.053 \\
\hline & & Glaze & 47.48 & 11.25 & 5.48 & 0.63 & 17.94 & 5.27 & 3 & 2.11 & 1.25 & 0.51 \\
\hline
\end{tabular}

for the determination and analysis of chemical composition of Shouzhou kiln yellow glaze porcelain: dl-01 fetal material: silica $66.51 \%$, aluminum oxide $25.2 \%$, and iron oxide $3.48 \%$; glaze: silicon dioxide $61.21 \%$, aluminum oxide $14.45 \%$, calcium oxide $14.52 \%$, and phosphorus pentoxide $1.33 \%$ and Dl-02 tire material: silica $68.33 \%$, aluminum oxide $24.3 \%$, and iron oxide $3.17 \%$; glaze: silica $59.23 \%$, aluminum oxide
$14.06 \%$, calcium oxide $15.15 \%$, and phosphorus pentoxide $1.28 \%$.

Systematically sort out the historical data of raw materials around Shouzhou kiln and establish the corresponding raw material database according to the chemical element content and composition data of various carcass raw materials. Specifically, it includes the following: first, the plasticity 
and fluidity test of ceramic slurry: the forming process of Shouzhou kiln includes the wheel forming process, grouting forming process, and rolling forming process. The corresponding mud data is established according to the methods and characteristics of Shouzhou kiln blank forming process; second, the temperature test of raw materials: the firing process of Shouzhou kiln has strict requirements on temperature, and the fire resistance of raw materials should reach $1300^{\circ} \mathrm{C}$. Therefore, a certain proportion of hightemperature-resistant raw materials should be added to the raw material formula of Shouzhou kiln and the relevant raw material temperature data should be established after the experiment; and thirdly, the glaze configuration technology of Shouzhou kiln: the key to the unique yellow glaze of Shouzhou kiln lies in the glaze formula technology. The glaze technology of Shouzhou kiln includes glaze classification, raw materials of glaze, glaze formula calculation, etc. According to the data of chemical element content and composition of raw materials in the glaze color of traditional Shouzhou kiln, the corresponding glaze database is established. The raw material database is input into the VR system developed by Shouzhou kiln ceramic products to realize that the material type, composition, and location can produce the final firing effect in combination with different firing temperatures and firing atmospheres. In the system, the designer can constantly try to replace the green body and glaze according to the green body thickness, fire resistance, glaze hardness, and glaze finish of the product and determine the final material ratio and combination from the final firing form reflected in VR.

\subsection{VR Technology Involved in Shouzhou Kiln Firing Process} Improvement. Ancient Shouzhou kiln had mastered advanced firing technology as early as the Sui Dynasty (581 618), and the glaze of ancient ceramic products excavated is still as bright as new. The reason why Shouzhou kiln has achieved such great success in history is mainly due to its early mastery of advanced firing technology and the glaze of utensils is fully melted, which is very suitable for daily life. What kind of atmosphere system is used to burn ceramic products? It shall be determined according to the composition of raw materials in the product formula and the physicochemical reaction in each stage of the firing process. When the raw material contains less organic matter and carbon, low viscosity, weak adsorption, and high iron content, it is suitable to choose the reducing atmosphere for sintering. On the contrary, it is suitable to choose the oxidation atmosphere. The firing atmosphere of ceramic products refers to the percentage of free oxygen and reducing components contained in the combustion products in the kiln during the firing process. Generally, the firing atmosphere is divided into oxidation atmosphere and reduction atmosphere. The atmosphere with free oxygen content above $8 \%$ is called a strong oxidation atmosphere. The atmosphere with free oxygen content of $4 \% \sim 5 \%$ is called an ordinary oxidation atmosphere. The atmosphere with a free oxygen content of $1 \% \sim 1.5 \%$ is called medium atmosphere. When the content of free oxygen is less than $1 \%$. And when the content of $\mathrm{CO}$ is less than $3 \%$, it is called a weak reduction atmosphere. The atmosphere with a CO content above $5 \%$ is called a strong reduction atmosphere. The content of iron in the glaze of Shouzhou kiln is high $(2.38 \% \sim 5.48 \%)$, and the content of titanium and manganese is low $(0.63 \% \sim 0.85 \%$ and $0.42 \% \sim 0.54 \%$, respectively), The main coloring element in the glaze is iron. Through the analysis of the ceramic fragments of ancient Shouzhou kiln, it is found that the content of coloring elements in the porcelain body and glaze of each sample is basically the same. It can be inferred that there are no additional coloring elements iron and titanium in the process of glaze making. The contents of coloring elements iron and titanium in the fetus and glaze of Guanzuizi green glaze sample and the yellow glaze sample of the inpatient department are basically the same. Therefore, the green glaze and yellow glaze are likely to be formed due to different firing atmospheres. The green glaze is formed in a reducing atmosphere and the yellow glaze is formed in an oxidizing atmosphere. The divalent iron ions in the green glaze are more than the yellow glaze, and the trivalent iron ions are less than the yellow glaze. Scholars speculate that the traditional firing method of Shouzhou kiln is oxidation, weak reduction, oxidation, strong reduction [11].

In the low-temperature stage of the firing process, the green body of Shouzhou kiln is mainly to remove water and carry out oxidative decomposition. In order to ensure the full oxidation of organic matter, carbon, and sulfide and the full decomposition of carbonate, a strong oxidation atmosphere is generally selected. In addition to the oxidation reaction described above, carbonate decomposition is also accompanied by ceramic products in the low-temperature stage of the firing process.

$$
\begin{aligned}
& \mathrm{MgCO}_{3} \mathrm{MgO}+\mathrm{CO}_{2} \uparrow\left(500 \sim 750^{\circ} \mathrm{C}\right), \\
& \mathrm{CaCO}_{3} \mathrm{CaO}+\mathrm{CO}_{2} \uparrow\left(550 \sim 1000^{\circ} \mathrm{C}\right) .
\end{aligned}
$$

The speed and completeness of these reactions are affected by the atmosphere. When the oxidizing atmosphere is suffcient, the reaction will be faster and more complete. On the contrary, the reaction speed becomes slow and incomplete. When the firing process enters the high-temperature stage, the gas produced by the liquid-phase reaction can not be freely discharged from the billet, so there are pinholes, bubbles, and other defects. It is impossible to oxidize and decompose all the gas components in the billet at the low-temperature stage, because carbonate and $\mathrm{Fe}_{2} \mathrm{O}_{3}$ decompose above $1300^{\circ} \mathrm{C}$ in an oxidizing atmosphere. However, in such a high-temperature region, the green body already has liquid a phase. When the viscosity decreases, the decomposed bubbles will break through the liquid phase and escape, resulting in uneven glaze or remaining in the glaze layer to form bubble defects. In order to solve this problem, the firing atmosphere should be controlled to a reducing atmosphere before high temperature (about $1000^{\circ} \mathrm{C}$ ), so that $\mathrm{Fe}_{2} \mathrm{O}_{3}$ and sulfate can undergo the following reduction decomposition:

$$
\begin{aligned}
& \mathrm{Fe}_{2} \mathrm{O}_{3}+\mathrm{CO}_{2} \mathrm{FeO}+\mathrm{CO}_{2} \uparrow, \\
& \mathrm{CaSO}_{4}+\mathrm{COCaSO}_{3}+\mathrm{CO}_{2} \uparrow, \\
& \mathrm{CaSO}_{3} \mathrm{CaO}+\mathrm{SO}_{2} \uparrow .
\end{aligned}
$$


The first reaction is strong at $1100^{\circ} \mathrm{C}$ in a reducing atmosphere. The latter reaction reacts strongly at $800^{\circ} \mathrm{C}$ in a reducing atmosphere. This ensures that such substances are fully reduced and decomposed before the glaze layer closes the green body [11]. Therefore, in order to eliminate the pinhole and bubble defects of Shouzhou kiln ceramic products, it is necessary to ensure that there is a strong oxidizing atmosphere in the preheating and a reducing atmosphere in the firing zone. Combined with multiple firing experiments, the firing atmosphere control of Shouzhou kiln can be adjusted as follows: strong oxidation $\left(960-1030^{\circ} \mathrm{C}\right)$-strong reduction $\left(1030-1280^{\circ} \mathrm{C}\right)$-weak reduction $\left(1280-1300^{\circ} \mathrm{C}\right)$.

VR ceramic firing simulation technology uses a computer to simulate a three-dimensional virtual visual traditional kiln, providing users with a visual, tactile, and auditory environment. Users can observe and control the ceramic products fired in the kiln from all angles without restrictions. Its fidelity and real-time interaction provide a strong support for the ceramic firing process of Shouzhou kiln. The main function of VR ceramic firing simulation technology is to truly simulate the whole process of ceramic molding, glaze configuration, firing system, and so on in the process of the traditional Jun porcelain intangible cultural heritage firing technology. It provides valuable reference data for the R \& D and innovation of contemporary Jun porcelain glaze.

Exploration and Application of Simulation Technology in the Production Process of Shouzhou Kiln. The simulation of Shouzhou kiln firing technology by virtual reality technology can not only shorten the R \& D cycle of Shouzhou kiln products and reduce $\mathrm{R} \& \mathrm{D}$ costs but also reduce the environmental pollution caused by long-term experiments. Using VR virtual reality, 3D glasses, and other auxiliary equipment, ceramic engineers can directly observe the realtime state of the glaze color in the firing process of Shouzhou kiln in the laboratory without going to the kiln workshop and find problems in the process of simulating temperature, reducing atmosphere, and glaze color, so as to timely and efficiently adjust various components of ceramic materials before mass production of ceramic products and accurately grasp the ceramic raw materials, temperature curve, reduction atmosphere data, product shrinkage ratio, and other data in the production of ceramics, so as to solve various problems found in $\mathrm{R} \& \mathrm{D}$.

The involvement of VR technology in the Shouzhou kiln firing process includes the following contents: first, fuel simulation: through the data analysis of chemical elements contained in firewood fired in Shouzhou kiln, the data analysis of the combustion value of various firewood, and the data analysis of metal substances contained in wood ash, the fuel database is established; second, temperature simulation: (1) temperature rise curve database: according to the characteristics of traditional firewood burning technology, analyze the temperature rise changes and firing time in each stage of the firing process and establish the corresponding firewood burning temperature curve data, (2) type of temperature sensor: firewood combustion is characterized by long flame, and the flame is in direct contact with utensils (naked combustion), so the contact temperature sensor is adopted, and
(3) number and distribution of temperature sensors: the number of temperature sensors is calculated according to the space size in the virtual Chai kiln, and the distributed structure is used for temperature sensing deployment in the kiln [12]; third, firing system simulation: (1) carbon dioxide database: according to the reduction reaction formula in the ceramic firing process $\left(\mathrm{Fe}_{2} \mathrm{O}_{3}+3 \mathrm{CO}=2 \mathrm{Fe}+3 \mathrm{CO}_{2}\right)$, analyze the $\mathrm{CO}_{2}$ content and distribution position in the reduction stage of Shouzhou kiln and establish the corresponding carbon dioxide concentration database an (2) furnace atmosphere pressure and flow rate database: test and analyze the atmosphere pressure and flow rate at different positions in the process of traditional Shouzhou kiln firing technology and establish the corresponding database.

3.4. VR Technology Involved in the Exhibition and Marketing of Shouzhou Kiln Ceramic Products. Build a VR display platform of Shouzhou kiln ceramic products based on e-commerce. With the continuous development of modern society, people's demand for ceramic products is gradually developing in the direction of multivariety and personalization. The design of traditional Shouzhou Kiln ceramics is generally formed in batches by ceramic craftsmen after making molds through grouting, extrusion, and other methods. Therefore, it is inevitable to have the following two shortcomings: first, the elements such as product shape and color are determined according to the craftsman's own aesthetic taste and cultivation. Therefore, the product characteristics are inevitably limited and it is difficult to realize multivariety and personalization. Second, it is impossible to establish communication channels with consumers, so it is somewhat blind in design, and it is difficult to flexibly adapt to the everchanging market demand. In view of this, with the help of VR technology, we can build a virtual Shouzhou kiln ceramic product display platform based on the e-commerce system, so that consumers can customize the shape, color, and other personalized elements of products according to their own needs without leaving home and truly experience the fun of customized products through interactive methods.

After completing the construction of the e-commerce website, it is necessary to refine the VR display platform of each virtual ceramic product. The steps are as follows and Figure 3:

Step 1. Construct the initial product model in 3D Max and other 3D software and convert it into a grid object (the number of points and surfaces should not be too large; otherwise, the subsequent operation will be affected), copy the initial model (keep the number of points and surfaces unchanged), and modify it into the end model $\mathrm{B}$.

Step 2. Assign materials and maps to the two models (UV coordinates must be specified to ensure correct model mapping). You can also choose the shell material in Virtools. The map is best refined with the unwrap UVW command to prevent aliasing when the model is changed in the future.

Step 3. Construct elements such as the background, environment, and lighting (the simpler the better). After the 


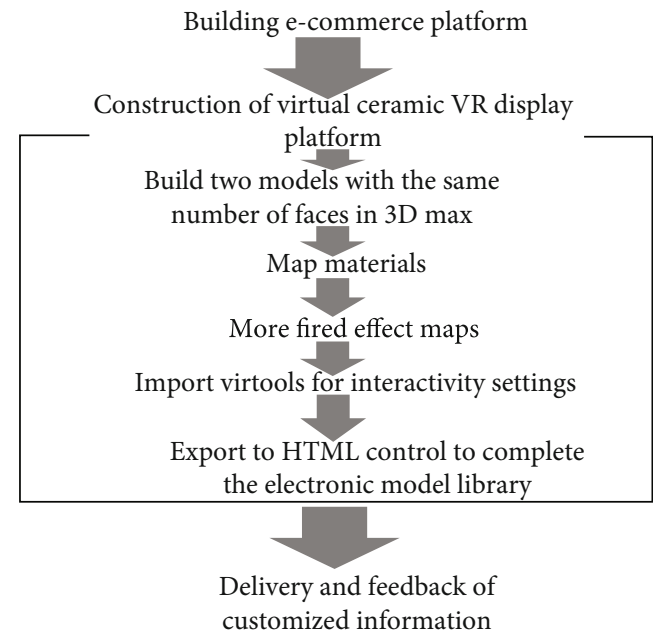

FIGURE 3: Construction flow chart of the Shouzhou kiln e-commerce system supported by VR technology.

rendering effect meets the requirements, use the "bake" command to generate the first set of colors, save the burned map, reassign its UV coordinates to the model, and render again to observe the effect. Change the map from the shader and use the bake command again to get the second set of maps. By analogy, multiple sets of baked maps are obtained.

Step 4. Import the generated model together with the baked map into the VIR tools software. You can add the shader provided by Virtools to debug and get the highlight and glaze effect of ceramics. Then, first formulate the basic operations such as zoom, translation, and rotation, and then, use the building block module to set two buttons, one to control the selection and replacement of colors (the thumbnail of each color can be placed below) and the other to control the change of the transition of the model from the initial state to the end point, which can be set into the form of the slider and digital display, The user can control the change of the product shape and record the numerical points through the slider.

Step 5. Complete other subsequent operations in Virtools and export them into HTML controls. Classify and sort out all the generated virtual ceramic product modules, use the website database to produce the electronic model library, and use ASP or PHP language to generate dynamic web page files to complete the production.

We are standing on the edge of a new communication era. The goal of information and communication technology (ICT) has developed from meeting the communication between people to realizing the connection between people and things, things, and things. The ubiquitous Internet of things communication era is coming. The Internet of things extends the traditional information and communication network to a wider physical world. Although the "Internet of things" is still a developing concept, bringing "things" into the "network" is a general trend of information development [13]. If VR is a virtual reality, the production of Shouzhou kiln is a complete reality. The necessary bridge between the two in line with the characteristics of information is the Internet of things technology. The combination of VR technology and the Internet of things provides a very broad development and imagination space for the product marketing and promotion of Shouzhou kiln. Using RFID technology as a substitute for bar code identification to realize the intelligent management of Shouzhou kiln product logistics system can realize the personalized customization of Shouzhou kiln ceramic products from users' high participation in design to blank forming and then to product firing, packaging, and logistics distribution, so as to realize the whole process real-time monitoring and users' participation in the whole process. The Internet of things is the "last mile" to realize the marketing of Shouzhou kiln products supported by VR technology.

\section{VR Technology Is Involved in the Inheritance of Shouzhou Kiln Technology and the Promotion of the Shouzhou Kiln Ceramic Culture}

Cultural promotion has become an important application field of VR technology. Now VR technology has become the core support technology of digital museums, science and technology museums, large-scale event opening and closing ceremony rehearsal simulation, digital museums, science and technology museums immersive interactive games and other application systems. It can be used for the recording, digitization and display of various documents, manuscripts, photos, movies, collections and other cultural relics. The high-precision modeling of these cultural relics also continuously puts forward higher requirements on VR modeling methods and data acquisition equipment, which promotes the development of VR. Many countries have actively carried out this work, such as the Metropolitan Museum in New York, the British Museum Russia's Winter Palace Museum, and France's Louvre which have established their own digital museums. China has also developed and established university digital museums, digital science and technology museums, and virtual Dunhuang and virtual Forbidden City [14].

As the dominant cultural and historical attribute, Shouzhou kiln must build a good cultural space and clear cultural identity in order to get a place in the current social space [15]. Combining traditional elements with modern aesthetics, while modern design injects vitality into the contemporary development of Shouzhou kiln, we must use effective scientific and technological means to realize the dissemination of Shouzhou kiln cultural identity. The core or main spiritual connotation of the living inheritance content of various traditional art and cultural activities is the collective aesthetic experience of specific cultural groups [16]. How to strike a balance between the two is an important issue in the development of Shouzhou kiln.

VR technology makes virtual scenes based on the real environment through the computer. Through VR technology, it can provide visitors with real-time interaction and realistic feeling, improve the speed of cultural relics display 


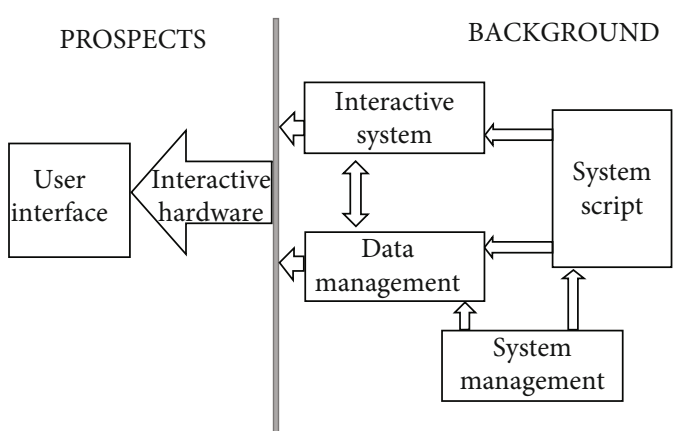

FIGURE 4: Shouzhou kiln VR display system structure.

and repair, reduce the use and operation cost, and provide a new idea for site display. As a world-famous category of ancient ceramics, Shouzhou kiln is not only an important production and export base of craft ceramics but also a window of the Chinese ceramic culture. At present, its display mode is mainly traditional entity, picture, and sample exhibition. It is a pity that this traditional display mode lacks interaction with tourists and can only provide static experience, but tourists spend time and energy but can not be satisfied. VR technology can well solve this problem and let visitors experience visiting the virtual space. By using VR technology, the exhibition can provide Shouzhou kiln with a virtual ceramic exhibition hall with friendly interaction and roaming functions. Customers can walk freely and watch freely in this virtual exhibition hall. At the same time, they can also observe more details of a single product with the help of interactive operation. The structure diagram of the Shouzhou kiln VR display system is shown in Figure 4. The system includes the user interface, interactive system, data management, system script, system management and other modules.

\section{Conclusion}

Culture is the sum of material civilization and spiritual civilization created by human beings. Shouzhou kiln is the crystallization of human spiritual and material creation and the materialized form of people's wisdom in the region. Technology is a method where people use existing things and knowledge to change the function and performance of existing things. VR technology is a bridge for human beings to shuttle freely between the imaginary space and the real space. The emergence of new technology will inevitably bring new development opportunities and space to the old cultural form. Similarly, the application of VR technology also brings new development opportunities for the ancient Shouzhou kiln. This opportunity comes from the comprehensive involvement of VR technology in Shouzhou kiln archaeology, Shouzhou kiln industry development, and Shouzhou kiln ceramic culture promotion. Technology and culture are never antagonistic. On the contrary, appropriate technology application can inject fresh vitality into the development of culture. With the unique 3I (immersion, interaction, and imagination) attribute of VR technology and the interconnection attribute of the Internet of things technology, the ancient Shouzhou kiln will have the opportunity to reenter the vision of today's people from the historical dust and glow with vigorous vitality. At the same time, VR technology's all-round involvement in the historical research, current development, and future inheritance of Shouzhou kiln also provides reference experience for the protection, development, and inheritance of human traditional ceramic culture.

\section{Data Availability}

Data sharing is not applicable to this article as no datasets were generated or analyzed during the current study.

\section{Conflicts of Interest}

The authors declared no potential conflicts of interest with respect to the research, authorship, and/or publication of this article.

\section{Acknowledgments}

The authors acknowledge Anhui Provincial Quality Engineering Project (2019jyxm0190), the scientific research project of Anhui University of Finance and Economics (ackyc20065), and Provincial Ideological and Political Demonstration Courses (2020szsfkc0014).

\section{References}

[1] Heim and J. Wulun, From Interface to Cyberspace: Metaphysics of Virtual Reality, L. Gang, Ed., Shanghai Science and Technology Education Press, Shanghai, 2000.

[2] I. Suther, "The ultimate display," in Proceedings of the International Federation of Information Congress, pp. 506-508, New York, 1965.

[3] M. Heim, Metaphysics of Virtual Reality, Oxford University Press, Oxford, 1993.

[4] G. Burdea and P. Coiffet, Virtual Reality Technology, John wiley and Sons, NJ, 1994.

[5] C. Wang, W. Gao, and X. Wang, The Theory, Implementation and Application of Lingjing (Virtual Reality) Technology, Qinghua University press, Beijing, 1996.

[6] Z. Qinping, H. Jinpeng, L. Bo, and S. Xukun, "Overview of virtual reality research," Computer Research and Development, vol. 33, no. 7, pp. 493-500, 1996.

[7] Z. Hu and L. Li, "Research and application of VR technology to the restoration and display of ancient ceramics," Information and Communication, vol. 3, 2014.

[8] L. Jun and G. Guohua, "3D realistic modeling and virtual display technology of cultural sites," Computer Engineering, vol. 36, 2010.

[9] S. Rangxiu, Qing Dynasty: Huaiyuan County Annals, wood movable type reprint, 1998.

[10] Y. Ruochun, Y. Zhengzheng, L. Yinghua, and C. Wang, "Test and preliminary analysis of Shouzhou kiln porcelain," Journal of China University of Science and Technology, vol. 1, pp. 2228, 2011. 
[11] H. Yundong, "Influence of firing atmosphere on defects of ceramic products and control analysis," Ceramics, vol. 6, pp. 33-35, 2002.

[12] P. Wang, "Application of orthogonal experimental method in the experiment of improving ceramic glaze hardness," Shandong Ceramics, vol. 4, pp. 22-23, 2013.

[13] International Telecommunication Union, Internet Reports 2005: The Internet of Things, ITU, Geneva, 2005.

[14] Z. Qinping, S. Xukun, and Q. Yue, "Research on some key technologies of digital museum," Journal of System Simulation, vol. 19, Supplement 2, pp. 1-6, 2007.

[15] Z. Yajun, X. Yu, and Z. Yongxi, "Development and thinking of Shouzhou kiln from the perspective of living inheritance," Journal of Liaoning University of Science and Technology, vol. 2, pp. 49-52, 2019.

[16] X. Gao, "Living protection of traditional art and construction of contemporary aesthetics," Literature and Art Research, vol. 7, pp. 5-13, 2013. 